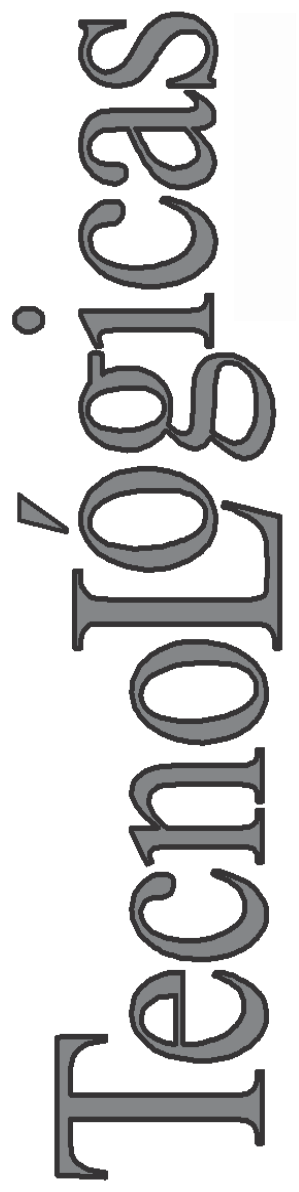

\title{
Evaluación de Emisiones Electromagnéticas Producidas por Hornos Microondas
}

\author{
Evaluation of Electromagnetic \\ Emissions Produced by Microwave \\ Ovens
}

Iván D. Vergara-Fuentes ${ }^{1}$ Henry A. Restrepo-Bohórquez ${ }^{2}$ Sara M. Yepes-Zuluaga ${ }^{3}$ Adolfo Escobar-Ordoñez ${ }^{4}$

1 Estudiante de Tecnología en Telecomunicaciones, Instituto Tecnológico Metropolitano, Medellín-Colombia ivanvergara3459@hotmail.com

2 Estudiante de Tecnología en Telecomunicaciones, Instituto Tecnológico Metropolitano, Medellín-Colombia jimhura@hotmail.com

3 Grupo de Investigación en Automática, Electrónica y

Ciencias Computacionales, Instituto Tecnológico Metropolitano, Medellín-Colombia sarayepes@itm.edu.co

4 Grupo de Investigación en Materiales Avanzados y

Energía, Instituto Tecnológico Metropolitano, Medellín-Colombia adolfoescobar@itm.edu.co 


\title{
Resumen
}

La preocupación pública por las fugas de los hornos microondas está presente desde el principio de su uso cuando muchas personas creían que dicha fuga era similar a la de la radiación atómica y que podría dar lugar a graves problemas de salud, incluyendo cáncer. En este artículo se presentan los resultados de la evaluación de emisiones electromagnéticas producidas por los hornos microondas que se encuentran en el área de cafeterías del ITM sede Fraternidad y que son usados por estudiantes, profesores y trabajadores para calentar sus alimentos. En primer lugar se hicieron mediciones a $5 \mathrm{~cm}$ de los hornos para identificar los máximos valores de fuga que presentaran los hornos. Posteriormente, se evaluó el nivel de campo en las mesas aledañas a los hornos para encontrar los niveles a los cuales estuvieran expuestas las personas que frecuentan la cafetería. Los resultados muestran que los máximos niveles encontrados no superan los límites establecidos en las normas internacionales ( $5 \mathrm{~mW} / \mathrm{cm} 2)$ e incluso no alcanzan el $1 \%$ de este valor.

\section{Palabras clave}

Emisión electromagnética; hornos microondas; fuga; alta frecuencia.

\begin{abstract}
Public concern about leakage from microwave ovens is present from the beginning of its use when many people believed that the leakage was similar to atomic radiation and could result in serious health problems, including cancer. This paper presents the results of the evaluation of electromagnetic emissions produced by microwave ovens that are in the area of meals of the ITM campus Fraternidad and are used by students, teachers and workers to heat food. First, measurements were made at 5 $\mathrm{cm}$ from the ovens to identify the maximum values of leakage generated by the ovens. Subsequently, it was evaluated the electric field level in the surrounding tables to the ovens to find the levels to which people to frequent the area were exposed. The results show that the highest level found does not exceed the limits set by international standards $(5 \mathrm{~mW} / \mathrm{cm} 2)$ and does not even reach $1 \%$ of this value.
\end{abstract}

\section{Keywords}

Electromagnetic emission; microwave ovens; leakage; high frequency. 


\section{INTRODUCCIÓN}

Un horno de microondas es un electrodoméstico que cocina o calienta alimentos por calentamiento dieléctrico. Esto se logra mediante el uso de radiación de microondas para calentar el agua y otras moléculas polarizadas dentro de la comida. Esta excitación es bastante uniforme, llevando a que la comida se caliente de manera más uniforme (excepto en objetos gruesos) de lo que generalmente ocurre en otras técnicas de cocción. Los hornos microondas básicos calientan los alimentos de manera rápida y eficiente, pero no hornean o doran la comida en la forma en que lo hacen los hornos convencionales.

La fuente de la radiación en un horno de microondas es el tubo de magnetrón, que convierte la corriente de alimentación del toma eléctrico a la radiación electromagnética a $2450 \mathrm{MHz}$ (para la mayoría de los hornos convencionales). El alto voltaje (aproximadamente 3 o $4 \mathrm{kV}$ ) que alimenta el tubo de magnetrón es producido por un transformador elevador rectificador, y un filtro que convierte el voltaje de red $\mathrm{AC}$ a $4 \mathrm{kV}$ DC. La energía de microondas desde el magnetrón se transfiere a la cavidad del horno a través de una sección de guía de onda. Una especie de agitador difunde la energía de microondas más o menos uniformemente por todo el horno.

Los hornos de microondas están fuertemente protegidos para que no tengan fugas y se apaguen automáticamente si se abre la puerta. El consenso general de las autoridades a nivel mundial con respecto a las fugas en los hornos microondas es que los niveles de fuga de los hornos en buen estado de funcionamiento son bajos y que no representan un peligro para la salud. Sin embargo, existen normas y estándares a nivel internacional que regulan la fuga de radiación permisible de los hornos de microondas. En Canadá, la Parte III (hornos de microondas) de la Radiation Emitting Devices Regulations (Minister of Justice, 2006) especifica los siguientes límites para la fuga de radiación a $5 \mathrm{~cm}$ de la superficie del horno: $1 \mathrm{~mW} / \mathrm{cm}^{2}$ con carga de prueba (50 ml de agua) y $5 \mathrm{~mW} / \mathrm{cm}^{2}$ sin carga de prueba. El estándar estadounidense (FDA, 1973) especifica un nivel máximo de emisión de $1 \mathrm{~mW} / \mathrm{cm}^{2}$ a $5 \mathrm{~cm}$ del horno antes de su adquisición por el comprador y no más de $5 \mathrm{~mW} / \mathrm{cm}^{2}$ durante su vida de operación. Normas similares se utilizan en 
otros países, algunos países también requieren que los hornos microondas sean revisados cada tres años. En Colombia, la norma NTC 4556 (ICONTEC, 2008) establece que la fuga de microondas en cualquier punto ubicado a $5 \mathrm{~cm}$ o más respecto de la superficie externa del artefacto no debe exceder de $5 \mathrm{~mW} / \mathrm{cm}^{2}$.

Se han realizado varios estudios sobre las fugas en hornos microondas. Un estudio (Skotte, 1981) examinó los hornos usados en restaurantes y cafeterías y encontró que para la mayoría de los grandes hornos la fuga está en el intervalo entre 0,2 a $2 \mathrm{~mW} / \mathrm{cm}^{2}$. Algunos hornos irradiaban más que el límite especificado y esto se atribuyó a la edad del horno y la falta de limpieza y mantenimiento. Otro estudio de emisión en hornos microondas se llevó a cabo en Alemania (Matthes, 1992), en el que se probaron 130 hornos. La potencia del horno oscilaba entre $350 \mathrm{~W}$ y $1200 \mathrm{~W}$ y la edad de los hornos entre 0,5 y 18 años. Las fugas medidas fueron inferiores a $1 \mathrm{~mW} / \mathrm{cm}^{2}$. El $50 \%$ de los hornos emitían menos de 0,062 $\mathrm{mW} / \mathrm{cm}^{2}$ y la probabilidad de emitir más de $5 \mathrm{~mW} / \mathrm{cm}^{2}$ era menor de 0,05\%. Otro estudio de 106 hornos microondas en Arabia Saudita (Alhekail, 2001) mostró que sólo un horno excedió el límite de emisiones de $5 \mathrm{~mW} / \mathrm{cm}^{2}$. Los estudios han encontrado que la radiación en la parte frontal es mayor debido a que la puerta del horno se encuentra ubicada en esta zona y que la radiación de la cara lateral izquierda, que está cerca del generador de microondas, ocupa el segundo lugar (Yinghong et al., 2003).

Un estudio más reciente (Muhammad Zin et al., 2011) realizó mediciones en 6 hornos encontrando que en un sólo horno microondas da un valor de $10,19 \mathrm{~mW} / \mathrm{cm} 2$ que supera el límite de $5 \mathrm{~mW} / \mathrm{cm}^{2}$. En otro estudio realizado en 117 hornos de uso doméstico y en restaurantes en Cisjordania-Palestina (Lahham \& Sharabati, 2013), con potencias entre 1000 y $1600 \mathrm{~W}$ y entre 1 y 20 meses de funcionamiento, se encontraron valores entre 0,43 a 16,4 $\mu \mathrm{W} / \mathrm{cm}^{2}$ con un valor promedio de $3,64 \mu \mathrm{W} / \mathrm{cm}^{2}$. La medida se llevó a cabo a una distancia de $1 \mathrm{~m}$ y a la altura del centro de la pantalla de la puerta de los hornos. En ningún caso la fuga excedía el límite de $1 \mathrm{~mW} / \mathrm{cm}^{2}$ recomendado por el ICNIRP.

Debido a que los hornos microondas se usan en la preparación de alimentos y en las áreas de cafetín a las cuales tienen acceso muchas personas, estos deben estar en buenas condiciones de 
funcionamiento para evitar que presenten fugas y supongan un riesgo para la salud de las personas. Debido a este problema, es importante llevar a cabo mediciones de fugas de energía electromagnética para los distintos hornos microondas que se encuentren disponibles en el mercado. Actualmente, la cafetería principal del Instituto Tecnológico Metropolitano sede Fraternidad, cuenta con 4 hornos a disposición de los estudiantes, profesores y trabajadores para calentar sus alimentos durante el transcurso del día. En este trabajo se presentan las mediciones de fuga de radiación en estos hornos y su distribución cerca de las mesas ubicadas en las cercanías de estos hornos.

\section{METOdOLOGía}

La medición de radiación electromagnética se hizo mediante el equipo Narda PMM8053B como sistema de adquisición de datos y la sonda EP 301 como elemento sensor. Este sensor tiene un rango de frecuencia de $0,1 \mathrm{MHz}$ a $3 \mathrm{GHz}$ y un rango de medición de 1 a $1000 \mathrm{~V} / \mathrm{m}$, soportando una sobrecarga de $1200 \mathrm{~V} / \mathrm{m}$. Igualmente, tiene una resolución de $0,1 \mathrm{~V} / \mathrm{m}$ y un error absoluto de $\pm 0.8 \mathrm{~dB}$.

Los hornos microondas medidos se encuentran ubicados en la cafetería principal. La cantidad de equipos ubicados en el área de la cafetería son 4 , dentro de los cuales dos de estos corresponden a una misma marca como se puede observar en la Fig. 1. Aunque la cafetería cuenta con 4 hornos microondas, durante el proceso de medida uno de los hornos (indicado en la Fig. 1) se encontraba en mal estado, por lo cual se realizaron las mediciones respectivas para los tres hornos restantes. Dos de los hornos medidos tenían una potencia nominal de $1200 \mathrm{~W}$ y el horno restante una potencia de $950 \mathrm{~W}$.

Para identificar la zona de los hornos microondas con mayor radiación de fuga se establecieron varios puntos de medida en la parte frontal y en los laterales de cada horno, tomando los puntos como referencia a estudios realizados en el artículo (Muhammad Zin et al., 2011) el cual establece que la mayor radiación de fuga en los hornos microondas se presenta en la parte frontal, en donde se encuentra ubicada la puerta. La Fig. 2, ilustra seis puntos de la 
cara frontal de uno de los hornos, en donde se encuentra ubicada la puerta, dentro de los cuales se realizó una medida correspondiente a cada punto. Donde h1 y h2 son las alturas de los puntos medidos con respecto al horno. Los valores de estos respectivamente son $10 \mathrm{~cm}$ y $20 \mathrm{~cm}$.

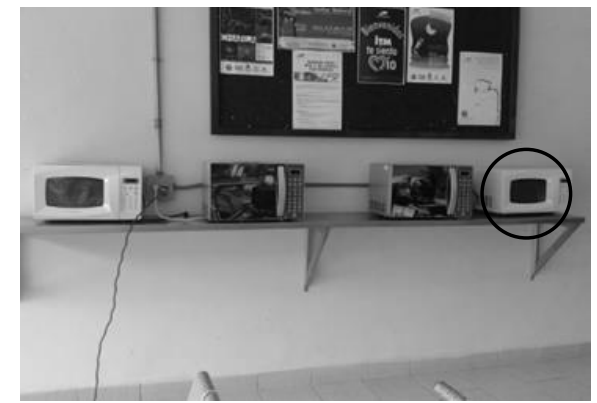

Fig. 1. Hornos microondas medidos. Fuente. Autores

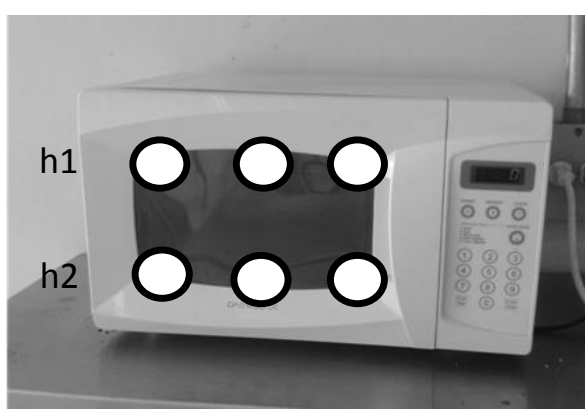

Fig. 2. Puntos medidos en los hornos. Fuente. Autores

Para iniciar la mediciones, se puso en funcionamiento el horno microondas con una carga de $275 \mathrm{~g} \pm 15 \mathrm{~g}$ de agua potable a $20^{\circ} \mathrm{C} \pm$ $2^{\circ} \mathrm{C}$, en un recipiente de pared delgada, hecho de vidrio de borosilicato, con un diámetro interno de aproximadamente $85 \mathrm{~mm}$. El horno se alimentó a la tensión nominal y se hizo funcionar estando el control de potencia del horno microondas en el ajuste máximo. Una vez ingresado el recipiente y de haber tenido en cuanta la distancia de medida recomendada por la norma, se procedió a iniciar la medida de los puntos en cada uno de los hornos microondas, como se muestra en la Fig. 3. 
La distancia a la cual se realizó la medida para los puntos anteriormente mencionados se tuvo en cuenta según la norma NTC 4556, ubicando el sensor a $5 \mathrm{~cm}$ del horno microondas. La fuga de microondas se determinó midiendo la intensidad de campo que en un término de 2 a 3 segundos alcanzara un $90 \%$ de su lectura en estado estacionario. El sensor se ubicó en los puntos descritos y adicionalmente se movió sobre la superficie externa del horno para localizar la máxima fuga de microondas, dedicando atención especial a la puerta y a sus cierres. Luego de realizar la medición se evaluaron los valores obtenidos, comparándolos con los indicados por la norma.

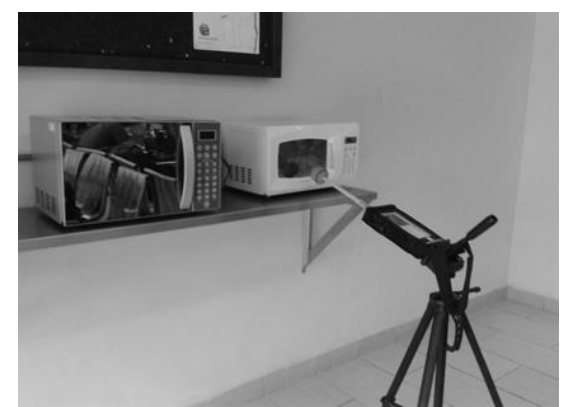

Fig. 3. Toma de medidas en un horno. Fuente. Autores

Por otro lado, se realizó la evaluación de la exposición a la radiación electromagnética producida por los hornos en el área de la cafetería donde estos se encuentran. La evaluación espacial del campo eléctrico se realizó en un área de $49 \mathrm{~m}^{2}$ en donde se tomaron en total 63 puntos de medida. Todas las medidas se hicieron a una altura de 1 metro sobre el nivel del suelo.

\section{RESULTADOS Y DISCUSIÓN}

En la Fig. 4 se observan los valores máximos medidos a una distancia de $5 \mathrm{~cm}$ para cada uno de los tres hornos en funcionamiento. Según los valores obtenidos en las mediciones, se identifica que la ubicación donde se presenta mayor radiación de fuga es en la parte frontal del horno, como se ha encontrado en otros estu- 
dios. El valor más alto medido correspondió a $0,437 \mathrm{~mW} / \mathrm{cm}^{2}$, lo que corresponde a menos del $1 \%$ de lo establecido en las normas nacionales e internacionales.

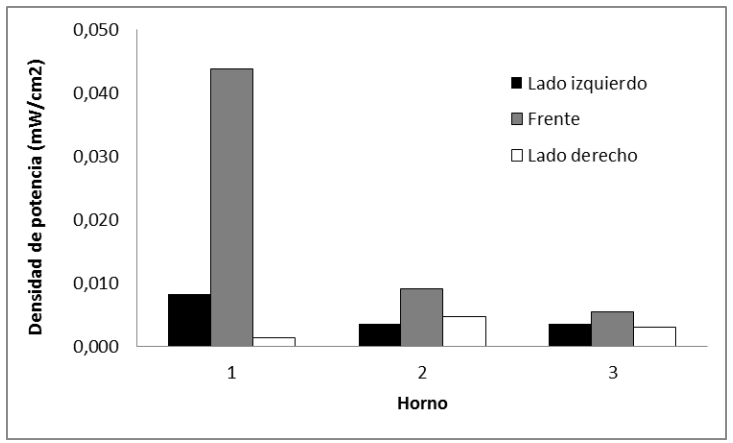

Fig. 4. Valores medidos a $5 \mathrm{~cm}$ de distancia de los hornos. Fuente Autores

Para la evaluación espacial de la fuga de radiación electromagnética producida por los hornos microondas se usó el software ViCo v1.0. Este software fue desarrollado en el marco del proyecto de investigación P10213 dentro del Instituto Tecnológico Metropolitano y tiene como fin visualizar intensidades de variables físicas interpoladas en un área específica. La elaboración de los mapas de contorno se llevó a cabo en la zona de la cafetería de la institución universitaria, en donde los estudiantes, profesores y trabajadores constantemente hacen uso de los hornos para la calefacción de sus alimentos. Antes de evaluar los hornos, se hizo una medición con estos apagados para conocer el ambiente electromagnético. Se asume que los valores obtenidos (máximo 1,01 V/m) son debidos a la interacción producida por otros sistemas como WiFi que operan a la misma frecuencia y que se sitúan dentro del espacio donde se realizaron las mediciones. En la Fig. 5 se muestra el mapa de contorno del campo eléctrico ambiente con un solo horno en funcionamiento. Por otro lado, en la Fig. 6 se observan los niveles de campo eléctrico medidos cuando se encontraban los tres hornos microondas funcionando simultáneamente. 


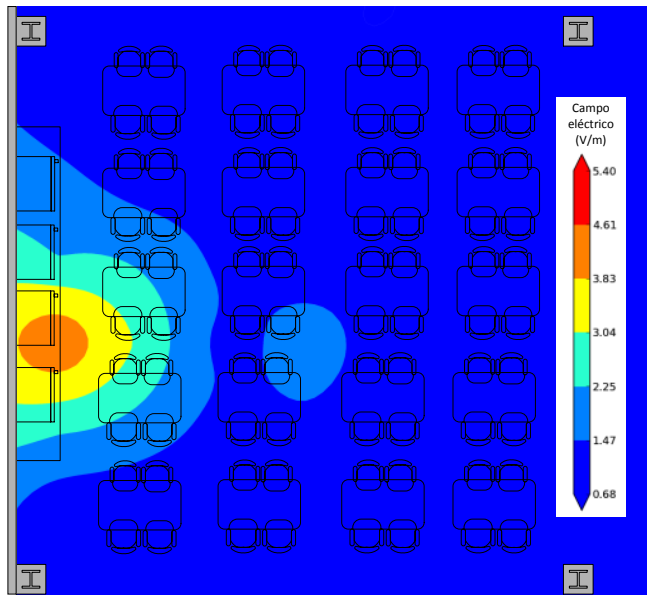

Fig. 5. Mapa de contorno con un horno funcionando. Fuente Autores

Se observa que el mayor nivel de campo medido fue de 5,4 V/m lo que corresponde a $0,00773 \mathrm{~mW} / \mathrm{cm}^{2}$ muy por debajo de los límites establecidos en normas internacionales para exposición de las personas (ICNIRP).

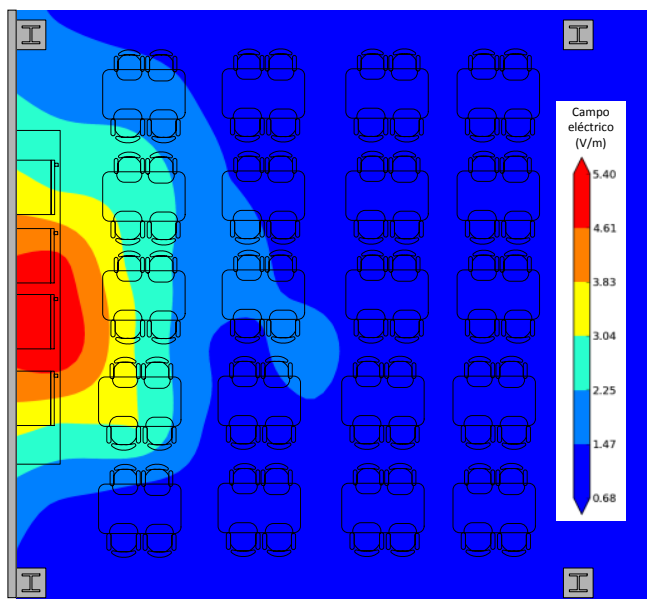

Fig. 6. Mapa de contorno con los hornos microondas funcionando. Fuente Autores 


\section{CONCLUSIONES}

Dentro de los valores máximos obtenidos para los tres hornos microondas se observa que no superan el umbral establecido por la norma, aunque algunos de estos valores sean mayores que otros debido a la posición en la cual se realizó la medida. Para cada horno en total se realizaron 8 medidas, 2 de las cuales corresponden a cada uno de los lados y 6 frontales dentro del cual se identificó la posición con mayor radiación y la cual a su vez fue tomada como referencia en las mediciones del mapa de contorno.

A pesar de que los valores obtenidos en las medidas no superan los establecidos, se observa a través de los mapas de contorno realizados en base a los datos que existe una radiación de fuga eminente por parte de los hornos microondas y se ilustra las zonas en las que la radiación es mayor. Con base en el criterio de operación a la cual trabajan los hornos, se observó la radiación existente aun cuando los hornos se encuentran apagados.

De igual forma e independiente del aporte que otros sistemas suministren en la toma de datos de las medidas, es posible observar en los mapas de contorno de los hornos en operación como se presenta un incremento en los valores obtenidos lo cual muestra indicios de la radiación inminente que estos proporcionan, de cómo los sectores con mayor radiación de fuga son los más cercanos a los hornos y la reducción en los datos a medida que la distancia de separación de los puntos medidos aumenta.

\section{REFERENCIAS}

Alhekail, Z. O. I. (2001). Electromagnetic radiation from microwave ovens. Journal of Radiological Protection, 21(3), 251-258. doi:10.1088/0952$4746 / 21 / 3 / 303$

FDA. Performance Standards for Microwave and Radio Frequency Emitting Products (1973). United States: U.S. Food and Drug Administration.

ICONTEC. Seguridad de artefactos electrodomésticos y artefactos eléctricos similares. Parte 2. Requisitos particulares para hornos microondas, incluyendo hornos microondas combinados (2008). Colombia: Icontec Internacional. 
Lahham, A., \& Sharabati, A. (2013). Radiofrequency radiation leakage from microwave ovens. Radiation protection dosimetry. doi:10.1093/rpd/nct173

Matthes, R. (1992). Radiation emission from microwave ovens. Journal of Radiological Protection, 12(3), 167-172. doi:10.1088/09524746/12/3/003

Minister of Justice. Radiation Emitting Devices Regulations (2006). Canada.

Muhammad Zin, N., Mohamed Jenu, M. Z., \& Ahmad Po'ad, F. (2011). Measurements and reduction of microwave oven electromagnetic leakage. In 2011 IEEE International RF \& Microwave Conference (pp. 1-4). IEEE. doi:10.1109/RFM.2011.6168681

Skotte, J. (1981). Undersoglese af mikrobolgeovne I storkokkener.

Yinghong, W., Linchang, Z., Chen, L., \& Xiaodong, Z. (2003). Measurement and calculation of the radiation characteristics of microwave ovens. In 2003 IEEE International Symposium on Electromagnetic Compatibility, 2003. EMC '03. (pp. 92-94 Vol.1). IEEE. doi:10.1109/ICSMC2.2003.1428201 